\title{
Stability of chronic multichannel neural recordings: Implications for a long-term neural interface
}

\author{
Justin C. Williams, Robert L. Rennaker, Daryl R. Kipke* \\ Bioengineering Program, Arizona State University, Tempe, AZ 85287-6006, USA
}

\begin{abstract}
Chronic multichannel neural recording has emerged as a powerful technique for studying dynamic brain function. In many experimental paradigms, it is important that the same neurons be recorded from day to day. The objective of this study was to develop methods for tracking the recorded neural population over time through analysis of unit waveforms, principle component clusters and response properties of single units and multiunit clusters. We demonstrate that these techniques can be used to provide a useful measure of unit stability over extended recording periods. (C) 1999 Published by Elsevier Science B.V. All rights reserved.
\end{abstract}

Keywords: Multichannel electrodes; Auditory cortex; Principle component analysis; Recording stability

\section{Introduction}

Long-term, multichannel neural recordings in behaving animals promise to revolutionize neuroscience by offering an unparalleled window on detailed brain responses over an extended time period. While this technique has been available for many years [10], only relatively recently have recording and multichannel electrode technologies advanced to the point of being used by many investigators in several neural systems (e.g., $[3,8,9])$. Although it is relatively common to record from an individual animal for several months, little has been reported describing the stability of the recordings: Specifically, to what extent is the same neural population recorded from day to day? Despite the caveat that the answer depends on the type of electrode, implantation

\footnotetext{
* Corresponding author.

E-mail address: kipke@asu.edu (D.R. Kipke)
} 
technique, and implant area (and, perhaps even the definition of a neural population), there remains a need to develop quantitative methods of addressing this question from chronic neural recordings.

Our research focus is on investigating the dynamics of long-term cortical plasticity [12] and the development of CNS-based neural prostheses [6]. These studies involve relatively simple microwire electrode arrays that are permanently implanted in the cortex to provide daily multichannel recordings from the same region of the brain $[1,11]$. The objective of this study was to develop methods for tracking the recorded neural population from day to day through the analysis of unit waveforms and response properties of the single units and multiunit clusters.

\section{Methods}

\subsection{Electrode implant}

Arrays of tungsten microwires were permanently implanted in auditory cortex of adult guinea pigs to provide simultaneous recordings of extracellular activity from a large number of cortical neurons over months. In the current experiments, the electrode arrays consisted of 33 wires arranged in a rectangular $3 \times 11$ grid. The $35 \mu \mathrm{m}$ tungsten wires were insulated to their tips with a $7 \mu \mathrm{m}$ thick layer of polyimide (California Fine Wire, Grover CA). The spacing between wires in a row was approximately $250 \mu \mathrm{m}$ apart, while the row spacing was approximately $350 \mu \mathrm{m}$. The electrode array terminated in a 33-channel sub-miniature connector. Under aseptic conditions and using standard stereotaxic surgical procedures, the array was slowly inserted into auditory cortex until the electrode tips were approximately $750-1000 \mu \mathrm{m}$ below the surface. The array was then permanently held in place by fixing the wire bundle to the skull using dental acrylic.

\subsection{Neural recording}

After a 2-3 day recovery period, simultaneous neural recordings were obtained from awake and behaving animals. The extracellular potentials from each wire were buffered using JFET amplifiers and then input to a multichannel neural recording system (Plexon, Inc., Dallas, TX). This system provided differential amplification, sampling (12 bit resolution, $40 \mathrm{kHz}$ sample rate), and channel-specific processing of the signals in order to store the waveforms and times-of-occurrence (spike times) of the single- and multi-unit activity from each channel. Units were discriminated by comparing amplitude-thresholded waveforms (typically $0.8 \mathrm{~ms}$ in duration) to one or more waveform templates (up to four templates per channel). At the beginning of each recording session, the experimenter adjusted the waveform templates and goodnessof-fit criterion for each template in order to qualitatively optimize the unit discrimination for each channel. Adjustment of amplitude thresholds above the noise level, online principle component analysis (see below) and standard auditory and visual cues were used to guide the unit discrimination process. 


\subsection{Analysis}

The spike waveforms for each channel were processed off-line to determine their variability from day-to-day over a period of months. Standard principle component analysis [2] was used to parameterize each waveform by its first three principle components. The mean and variance for the principle component cluster for each unit were computed for each recording day. The spike properties of each unit were summarized using inter-spike interval histograms, peri-stimulus histograms, and frequency response areas, as described by Witte et al. [11].

\section{Results}

The data reported here were obtained as part of several ongoing experiments in which various simple auditory stimuli were used to characterize unit response properties in auditory cortex. Responses from three animals illustrate the basic methods for tracking unit stability over the duration of each implant (up to six months). A typical daily recording session involved recording from 30 to 70 single units and multiunit clusters, (one to three units were detected from each active electrode).

A well-isolated single unit from one animal was selected from a single electrode to examine waveform stability over a six week period. Fig. 1A shows a selection of spike waveforms from this unit, designated $13 \mathrm{a}$, that over this time span illustrates the typical variability in the recorded waveform signal, both within one recording session and from day to day. This unit is an example of what can be considered a stable unit, one whose waveform shape does not vary considerably and that can be distinctly identified from one day to the next.

From the same set of waveforms, the first three principle components were computed and the mean and standard deviation of each component was calculated for each recording session. These values were used to plot an ellipse for each day whose center is at the mean of the principle components and whose radius is equal to one standard deviation. Ellipses were plotted for each recording session and were fit with a three-dimensional surface mesh to form a Principle Component Stability Tube, or PCST. Fig. 1B illustrates the PCST for the unit corresponding to Fig. 1A. Since the spike waveforms were discriminated with a waveform template that was selected to optimize this single-unit discrimination, the PCST, in this case, indicates how the template was adjusted each day to best discriminate the unit waveforms on that day. In this sense it can be thought of as a quantitative measure of the qualitative assessments made by the user.

Basic spike firing statistics were computed for this unit in order to relate waveform variability with response variability. The IHs were computed for each recording session in the six week period of analysis. For unit 13a, these response properties remained stable over the one month period, as shown for the IHs for three selected days (Fig. 1C). The stability of the IH supports the argument that unit 13a is the same neuron on each of the recording days. 

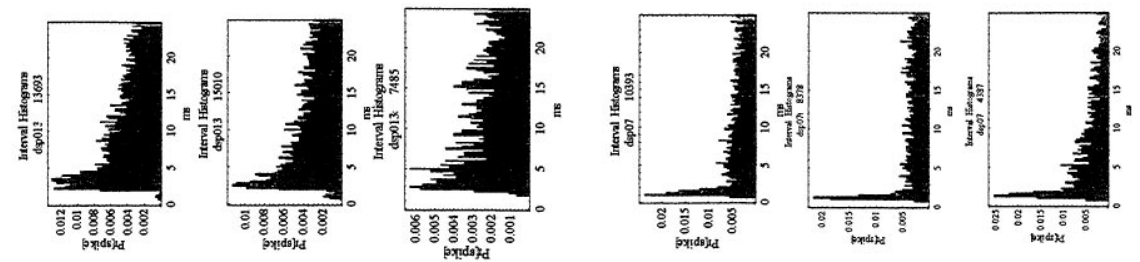

0
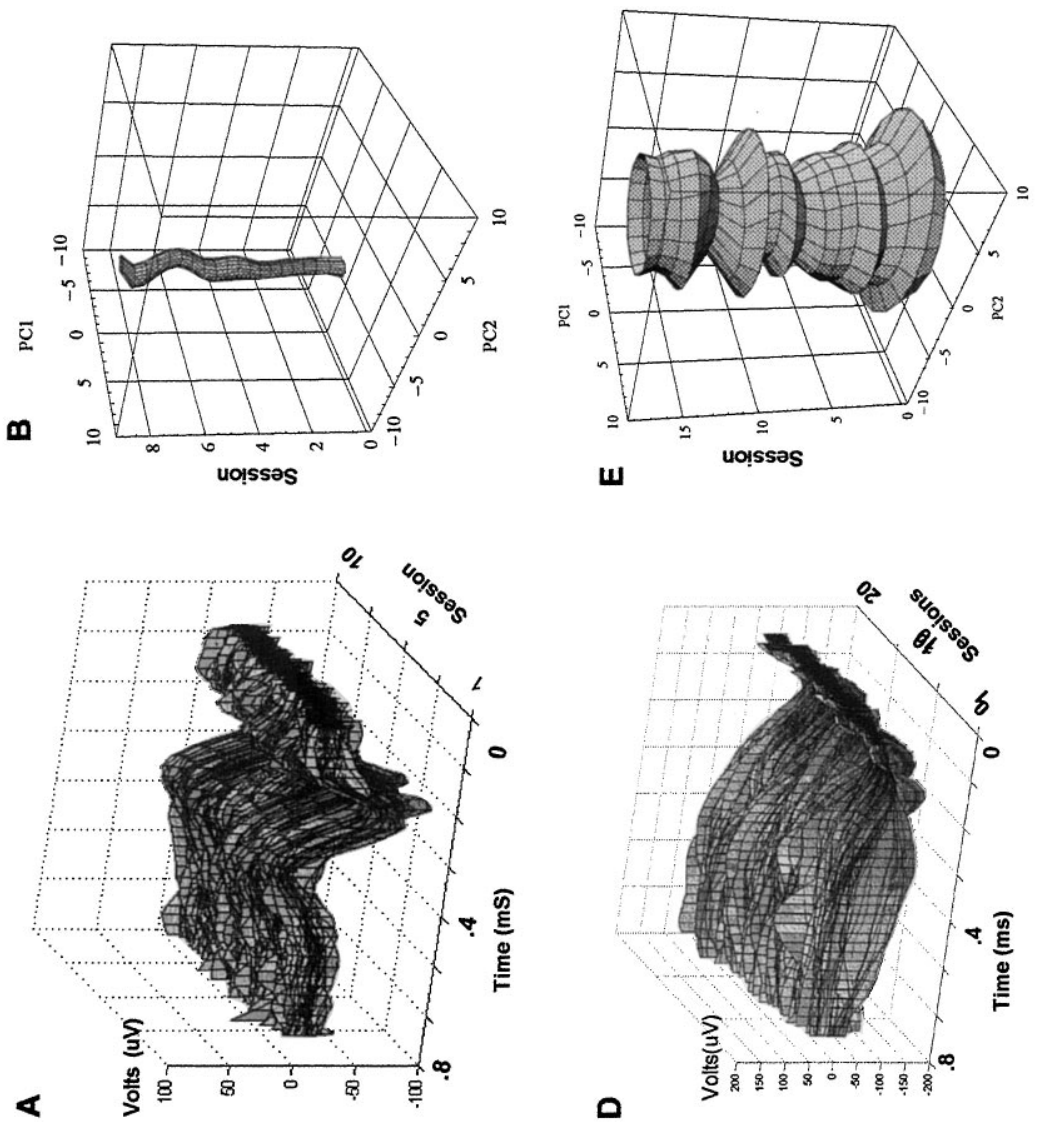


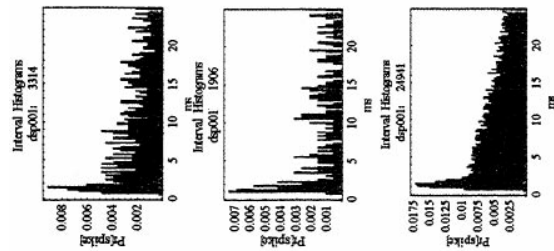

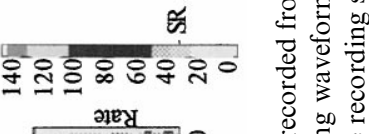

苛苾

घี

*
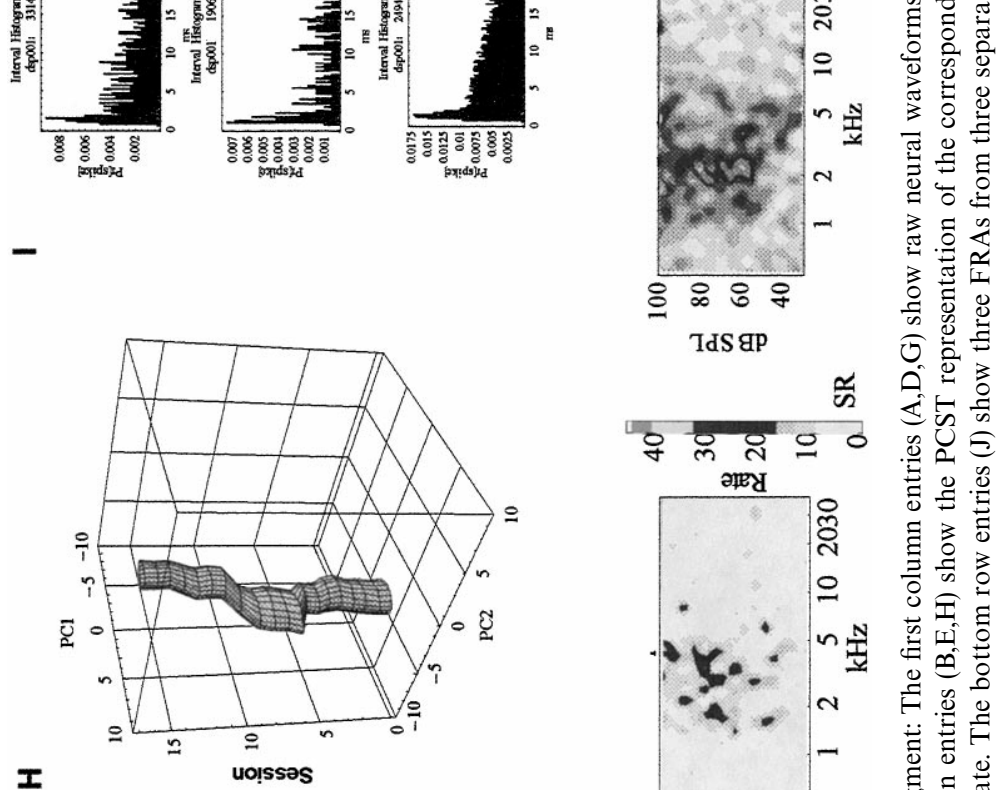

I

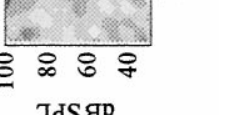

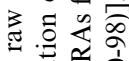

氙近

它节范

(0)

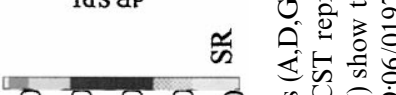

प

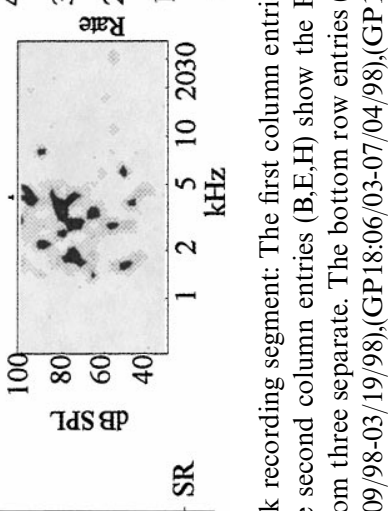

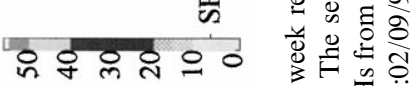

गएप

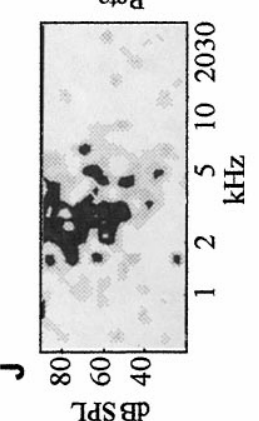

它声它

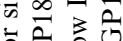

过过

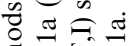

昰记范

E

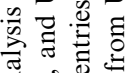

สี

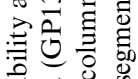

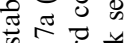

कृ.

$\exists \square$

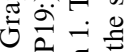

-

为焉焉 
The same techniques were used to analyze recordings of a multi-unit cluster that was consistently recorded on a single electrode for a six week period. Fig. 1D and E show this waveforms and PCST for the multi-unit cluster, designated unit 7a. The center of the PCST remained stable, but its large variance reflects the typical recording properties of low-amplitude multi-unit signals. This is in direct contrast to the fine discrimination and correspondingly slim PCST for the well-isolated single unit. In this case, unit discrimination was based mainly on amplitude thresholds so that the PCST reflects statistical variation in the waveforms rather than template selection. IHs for this unit showed variabilities consistent with a multiunit cluster (Fig. 1F).

A unit's responses to stimuli may provide additional information for assessing recording stability. Fig. $1 \mathrm{G}-\mathrm{J}$ summarizes a set of response properties for a second well-isolated and stable single unit. The frequency response areas (FRA), a measure of the unit's frequency selectivity [11], show consistent frequency selectivity during the time of interest. In this case, the FRA supports this being a stable unit although the corresponding IHs show some variability.

\section{Discussion}

In order to track the stability of recorded neural populations over months, we have analyzed spike waveforms and basic response properties. These measures suggest that periods of unit stability of at least six months are obtainable on at least some of the microwire electrodes. The PCST is useful for identifying trends and variability in waveshape properties through reducing thousands of sampled waveforms to just four numbers: the mean and variance in each of the two principle components. At present, we qualitatively assess the shape of the PCST and the supplementary response properties in order to gain some understanding of the types of responses that can be expected with these implants. In doing so, care must be taken to ensure that a large sample of waveforms is obtained by each recording session and that waveform discrimination criteria remain consistent from day to day. In any case, this approach provides a framework for developing more quantitative and closed-loop estimates of recording stability.

The importance of recording stability, and the precision at which it must be determined, depends on the experiment and or application of the chronic recordings. For example, neuroprosthetic applications will have different requirements than detailed connectivity experiments. Our current series of implants, with the relatively simple microwire electrode array provide a reference mark by which the recording performance of other types of implantable electrode systems can be evaluated $[4,5,7]$.

\section{References}

[1] R.S. Clement, R.S. Witte, R.L. Rennaker, D.R. Kipke, Functional connectivity in auditory cortex using chronic, multichannel microelectrodes in awake animals, Neurocomputing (1999), in press. 
[2] M.S. Fee, P.P. Mitra, D. Kleinfeld, Variability of extracellular spike waveforms of cortical neurons, J. Neurophysiol 76 (1996) 3823-3833.

[3] C.M. Gray, P.E. Maldonado, M. Wilson, B. McNaughton, Tetrodes markedly improve the reliability and yield of multiple single- unit isolation from multi-unit recordings in cat striate cortex, J. Neurosci. Methods 63 (1995) 43-54.

[4] A.C. Hoogerwerf, K.D. Wise, A three-dimensional microelectrode array for chronic neural recording, IEEE Trans. Biomed. Eng. 41 (1994) 1136-1146.

[5] D.T. Kewley, M.D. Hills, D.A. Borkholder, I.E. Opris, N.I. Maluf, C.W. Storment, J.M. Bower, K. Kovacs, Plasma-etched neural probes, Sensors Actuators A 58 (1998) 27-35.

[6] D.R. Kipke, P.D. Perepelkin, A.B. Schwartz, Chronic multiunit recording in monkey motor cortex: Implications for control of a motor prosthesis, Ann. Biomed. Eng. 24 (1996) 68.

[7] E.M. Maynard, C.T. Nordhausen, R.A. Normann, The Utah intracortical electrode array: a recording structure for potential brain-computer interfaces, Electroencephalogr. Clin. Neurophysiol. 102 (1997) 228-239.

[8] M.A. Nicolelis, A.A. Ghazanfar, B.M. Faggin, S. Votaw, L.M. Oliveira, Reconstructing the engram: simultaneous, multisite, many single neuron recordings, Neuron 18 (1997) 529-537.

[9] P.J. Rousche, R.A. Normann, Chronic recording capability of the Utah intracortical electrode array in cat sensory cortex, J. Neurosci. Meth. 82 (1) (1998) 1-15.

[10] E.M. Schmidt, M.J. Bak, J.S. McIntosh, Long-term chronic recording from cortical neurons, Exp. Neurol. 52 (1976) 496-506.

[11] R.S. Witte, K.J. Otto, J.C. Williams, D.R. Kipke, Pursuing dynamic reorganization in auditory cortex using chronic, multichannel unit recordings in awake, behaving cats, Neurocomputing (1999), in press.

[12] R.S. Witte, J.C. Williams, D.R. Kipke, Investigation of dynamic reorganization in auditory cortex using chronic multichannel neural recordings, Assoc. Res. Otolaryngol. 21 (1998) 148.

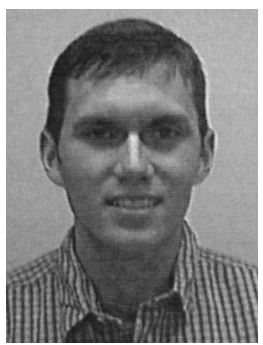

J.C. Williams received B.S. degrees in Mechanical Engineering and Physics from South Dakota State University in 1995 and 1996. He is currently a Ph.D. candidate in the Bioengineering Program at Arizona State University. His doctoral thesis concerns the characterization of chronic neural interface properties. His research interests are in the areas of implantable neural devices and instrumentation.

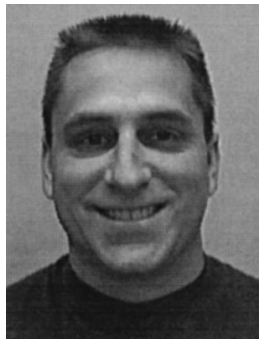

R.L. Rennaker received a B.S. degree in Biomedical Engineering from Arizona State University in 1997, and is currently a Ph.D. candidate in the same program. His research emphasis involves neural implant technologies and auditory physiology. 


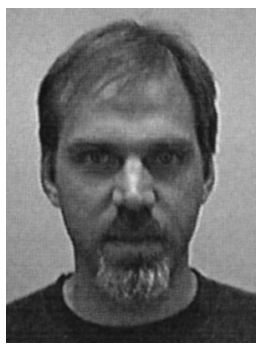

D.R. Kipke received a Ph.D. degree in Bioengineering from the University of Michigan in 1991. He is currently an Associate Professor in Bioengineering at Arizona State University. His research involves auditory processing, neural prostheses, cortical plasticity, and neural implant technologies. 